\title{
Ein neuer Tempel Salomons in Jerusalem? Der Bau der Nea-Kirche (531-543) durch Kaiser Justinian*
}

\begin{abstract}
The Roman Emperor Justinian (reg. 527-565) reshaped the urban structure of Jerusalem especially by expanding the cardo maximus and by the construction of a new Church of Mary, the so-called Nea. What might have been his intention, and what symbolic and theological messages did Justinian associate with the building of the Nea in Jerusalem? In this paper I consider the theory that Justinian wanted his church to outdo the Jewish Temple: accordingly, he intended the Nea to be not only a new church of Mary in Jerusalem, but also to a certain extent a new and better Temple of Solomon.

After sketching a brief overview of Justinian's building activity in the Holy Land I discuss the literary and archeological evidence regarding the Nea itself. Indications or arguments to support my assumption come from topography, archeology, literature, and especially from the Jerusalem liturgy. Unlike almost all other churches in Jerusalem the Nea was not directly connected to any biblical site. A connection to the events of the Gospels could be created by integrating the church liturgically into the calendar of feast days in Jerusalem. This was accomplished by tying the dedication date of the Nea to "the presentation of Mary". During this feast, the Mother of God was presumably celebrated in hymns as vaò $\varepsilon$ ع $\mu$ uxoৎ and by similar expressions. We thus may confirm that the Nea towered over the Temple of Solomon not only physically: as its liturgical integration into the church calendar makes clear, since it was dedicated to the Mother of God (theotokos) as the true, genuine Temple of God, it also surpassed the Jewish Temple theologically.

This concept applied by Justinian may also explain why the Nea had largely vanished from the scene already in the tenth century: since the Nea was not tied to a lieu de mémoire in a narrow sense, but rather derived its significance from an artificial theological concept which lost its plausibility after the Muslim rebuilding of the Temple mount, it could vanish from the sacred topography of Jerusalem unmourned and unsung - despite its former monumentality and liturgical prominence.
\end{abstract}

\footnotetext{
* Großen Dank weiß ich meinem Freund Michael Chronz (Bonn), der mich vor vielen Jahren auf das Thema aufmerksam gemacht und mir wertvolle Hinweise gegeben hat. Ebenfalls danke ich den Herausgebern Hartmut Leppin und Wolfram Brandes (beide Frankfurt a.M.) für ihre Verbesserungsvorschläge. Sofern nicht anders angegeben, stammen die verwendeten Übersetzungen vom Verfasser. Für Prokops „Bauten“ habe ich die Übersetzung von Otto Veh (München 1977) verwendet.
} 
Kaiser Justinian (reg. 527-565) hat mit seinen Bauten nicht nur Konstantinopel, sondern auch Jerusalem grundlegend verändert. In Jerusalem hat Justinian vor allem mit dem Ausbau des Cardo maximus und der Errichtung einer neuen Marienkirche, der sogenannten Nea, in die urbane Struktur eingegriffen. Über diese Baumaßnahmen, die sich im Wesentlichen über einen Zeitraum von zwölf Jahren, von 531 bis 543, erstreckten, berichten annähernd gleichzeitig und unabhängig voneinander zwei zeitgenössische Autoren und Augenzeugen sehr unterschiedlichen intellektuellen Formats: der Historiker Prokop von Kaisareia und der Hagiograph Kyrill von Skythopolis. $\mathrm{Zu}$ diesen literarischen Zeugnissen treten jetzt archäologische Befunde, denn der Teil Jerusalems, in dem die genannten justinianischen Bauten standen, ist von 1969 bis 1982 durch israelische Archäologen unter Leitung von Nahman Avigad gründlich erforscht worden. Der für mein Thema relevante Teil ihrer Forschungen wurde 2012 als Band V der Jewish Quarter Excavations in the Old City of Jerusalem von Oren Gutfeld publiziert. In dem folgenden Beitrag werde ich die Aussagen Prokops und Kyrills über die Nea diskutieren und mit den Ergebnissen der archäologischen Forschung konfrontieren (Abschnitt II). Auf dieser Basis kann gefragt werden, welche symbolischen und theologischen Botschaften Kaiser Justinian mit dem Bau der Nea in Jerusalem verband (Abschnitt III). Dabei wird die These erörtert, daß Justinian seine Kirche in eine typologische Nachfolge zu dem jüdischen Tempel stellt: Demnach ist die Nea nicht nur die neue Marienkirche Jerusalems, sondern in gewisser Weise auch der neue Tempel Salomons. ${ }^{1}$ Zunächst aber soll durch einen kurzen Überblick über die Bautätigkeit Justinians im Heiligen Land der Rahmen skizziert werden, in dem sich die Jerusalemer Aktivitäten des Kaisers vollzogen (Abschnitt I).

Die Regierungszeit des Kaisers Justinian (527-565) kann als Höhepunkt der spätantiken sakralen Bautätigkeit in Palästina und besonders in Jerusalem angesehen werden: Der Kaiser selbst gab Geld, wie der Historiker Prokop in den Bauten im Einzelnen auflistet, für Klöster, Kirchen, Armenhäuser, Herbergen, Zisternen und Brunnen, aber auch für den Bau von Festungen zum Schutz der Mönche und Pilger vor Wüstennomaden. Darüber hinaus investierte er in vier Großprojekte, von denen drei durch Prokop näher beschrieben worden sind: der Bau des Cardo maximus und der Nea-

1 Die These ist nicht neu, sondern wurde mit teils unterschiedlichen Argumenten, die in diesem Beitrag einer Prüfung unterzogen werden, vertreten bzw. erwogen von: H. Amitzur, Justinian's Solomons's Temple in Jerusalem, in: M. Poorthuis - Ch. Safrai (eds.), The Centrality of Jerusalem. Historical Perspectives, Kampen 1996, 160-175; I. Shahid, Justinian and the Christianization of Palestine, in: E. Evangelatou-Notara - T. Maniati Kokkina (eds.), Kletorion eis mnemen Nikou Oikonomide, Athen 2005, 373-385; J. Taylor, „The Nea Church“, Biblical Archaeology Review, 34.1, 2008, 51-59; O. Gutfeld et al., Jewish Quarter Excavations in the Old City of Jerusalem conducted by Nahman Avigad, 1969 - 1982, Volume V: The Cardo (Area X) and the Nea Church (Areas D and T). Final Report, Jerusalem 2012, hier 491- 494. 
Kirche in Jerusalem; die Befestigung der von Zenon erbauten Theotokos-Kirche auf dem Berg Garizim in Samaria durch eine massive Mauer, die Schutz vor samaritanischen Übergriffen bieten sollte; der Bau einer Kirche auf dem Moses-Berg im Sinai sowie eines Kastells zu Füßen des Berges; und (von Prokop nicht berücksichtigt, vielleicht weil das Projekt erst nach der Abfassung der Bauten realisiert wurde) die Erneuerung und Erweiterung der Geburtskirche in Bethlehem. ${ }^{2}$ Diese Liste evoziert sofort die Frage, warum der Kaiser so viele, so große und kostspielige Projekte in Palästina realisiert hat. Was war Justinians Interesse an Jerusalem und dem heiligen Land?

Zwei Antworten drängen sich auf: Zum einen war Palästina für die Kirchenpolitik des Kaisers von geradezu schicksalhafter Bedeutung. Anders als in Ägypten und Syrien hatte sich in Palästina nach anfänglichem Widerstand die Glaubensdefinition von Chalkedon weitgehend durchgesetzt. Ihr Festhalten an Chalkedon hatten die Patriarchen von Jerusalem und insbesondere die Mönche der judäischen Wüste mit großem Einsatz gegen die Interventionsversuche des antichalkedonensischen Kaisers Anastasios verteidigt. Nach der Machtübernahme des Kaisers Justin 518, der Chalkedon aufs Neue zur dogmatischen Richtschnur machte, wurde das Heilige Land daher sozusagen automatisch zur Bastion der kaiserlichen Kirchenpolitik im Orient. Für die Versuche Justinians, die Einheit der Kirche wiederherzustellen und eine für alle Seiten akzeptable Kompromißformel $\mathrm{zu}$ finden, spielte die Kirche von Jerusalem eine herausragende Rolle. An den entsprechenden Verhandlungen nahmen Bischöfe und vor allem Mönche aus Palästina in führender Position teil. Umgekehrt schaltete sich der Kaiser höchstpersönlich sowohl mit theologischen Traktaten als auch durch staatliche Machtmittel ein, als die Einheit der palästinischen Kirche in den späten dreißiger und frühen vierziger Jahren des 6. Jahrhunderts durch die sogenannten Origenisten in Frage gestellt wurde. ${ }^{3}$

Außerdem war es für den Kaiser natürlich auch wegen der vielen Pilger, die aus allen Reichsteilen zu den heiligen Stätten strömten, wichtig, im Einvernehmen mit den lokalen Autoritäten zu stehen. Auf diese Weise eröffnete sich dem Kaiser eine besonders wirkungsvolle Bühne der Repräsentation. Kaiser Justinian hat diese Bühne genutzt und die Idee vom Heiligen Land zum tragenden Bestandteil seines Selbstverständnisses gemacht. In einer Novelle aus dem Jahr 536 rühmt Justinian Palästina als Provinz ehrwürdiger Städte, guter und gelehrter Bürger und berühmter Gottes-

2 Prok. aed. 5,6-9; G.T. Armstrong, Fifth and Sixth Century Church Buildings in the Holy Land, Greek Orthodox Theological Review 14, 1969, 17-30, hier 23-28; zur Geburtskirche in Bethlehem vgl. M. Avi-Yonah, in: The New Encyclopedia of Archaeological Excavations in the Holy Land, Jerusalem 1994, vol. 1, 205-208; vgl. A. Cameron, Procopius and the Sixth Century, London 1985, 94-98, bes. 95 mit Anm. 88 .

3 Vgl. K. Trampedach, Reichsmönchtum? Das politische Selbstverständnis der Mönche Palästinas im 6. Jahrhundert und die historische Methode des Kyrill von Skythopolis, in: Millenium 2, 2005, 271 296, hier: $273-279.284$. 
männer und, „was das Größte von Allem ist“, als Ort der Erscheinung Christi. ${ }^{4}$ Eine weitere Verordnung aus dem gleichen Jahr erteilte der Kirche von Jerusalem wichtige finanzielle Privilegien. Zur Begründung führte der Kaiser unter anderem Folgendes an: „Wenn nämlich Gott, der Herr und zugleich Schöpfer von allem, sie [die Stadt Jerusalem] einer so großen Vergünstigung vor den anderen Städten gewürdigt hat, daß er dort nach dem Fleische wieder auferstanden ist, so ist offenkundig, daß auch wir, die wir dem Herrn und Gott und seinen großen Wundertaten, soweit menschenmöglich, nachfolgen, der dortigen Kirche vor den anderen Vergünstigungen gewähren: Daher wird sie dieses Gesetzes von uns teilhaftig, indem wir sie durch den Vorteil vor allen auswählen und ehren." ${ }^{5}$ Zuwendungen für Jerusalem gehören zum ideologischen Selbstverständnis des Kaisers; sie sind, wie Justinian selbst betont, Ausdruck jener imitatio dei oder mimesis theou, der sich der Kaiser besonders verpflichtet fühlte. Wie vor ihm nur Konstantin im 4. und, mit Abstrichen, die Kaiserin Eudokia im 5. Jahrhundert, hat denn auch Justinian das Heilige Land einer sehr intensiven Bautätigkeit gewürdigt - einer Bautätigkeit, die selbst bei diesem bauwütigen Kaiser außerhalb von Konstantinopel und abgesehen von den Militäranlagen entlang der Ostgrenze des Reiches ihresgleichen sucht. ${ }^{6}$

Unter den justinianischen Bauprojekten im Heiligen Landes waren zweifellos diejenigen in Jerusalem am bedeutendsten, und zwar nicht nur des Ortes wegen; der Aufwand, den Kaiser Justinian betreiben mußte, um den Cardo maximus auszubauen und die Nea-Kirche zu errichten, war um ein vielfaches größer als bei anderen Bauten im Heiligen Land. Einen besonders anschaulichen Eindruck des zeitgenössischen Jerusalem vermittelt die Mosaik-Karte von Madaba, die in der zweiten Hälfte des 6. Jahrhunderts geschaffen wurde. Von Jerusalem zeigt die Karte ein erstaunlich ge-

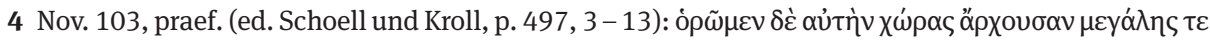

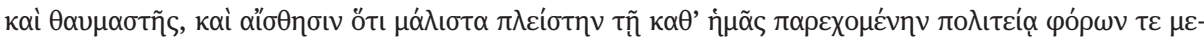

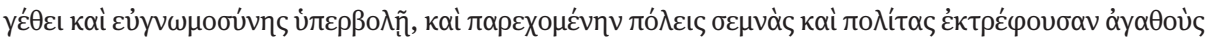

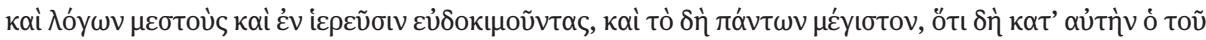

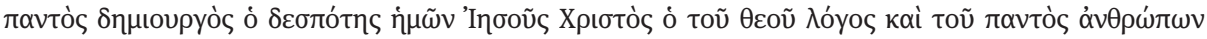

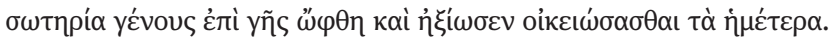

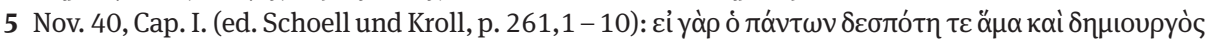

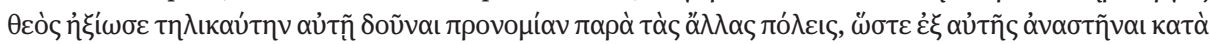

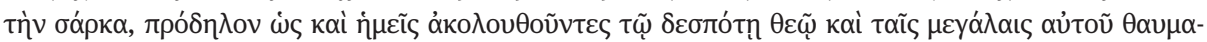

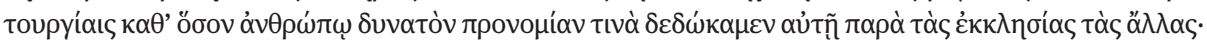

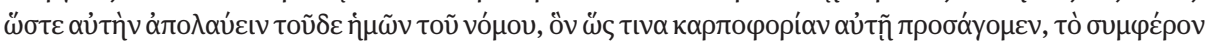

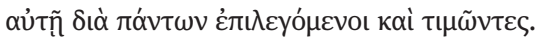

6 Alle vier genannten Großbauprojekte waren überdies durch die Besetzung entsprechender symbolischer Orte besonders geeignet, den Prozeß der „Dejudaisierung“ Palästinas im Zeichen des Christentums zu fördern - ein Ziel, das ohne weiteres an die antijüdische Gesetzgebung Justinians anschließt. Vgl. Shahid (s. Anm. 1) 374: „It is only natural for an emperor of such anti-Jewish cast of mind to direct his attention to Palestine in order to convert it from a Jewish Promised Land to a Christian Holy Land." In diesem Sinne überzeugend interpretiert Shahid 382 - 384, insbesondere das Prooimion der Nov. 103 (s.o.), in dem der Kaiser nicht nur Jesus Christus, sondern auch die Kaiser Vespasian und Titus in den höchsten Tönen rühmt. 
naues Abbild, wenn man von zwei bezeichnenden theologisch-ideologischen Verzerrungen absieht: a) Der Anastasis-Komplex befindet sich exakt auf der Querachse der Stadtelipse und wurde damit von den Schöpfern des Mosaiks ebenso in das Zentrum des Bildes gerückt wie die heilige Stadt insgesamt als Nabel der Welt in der Karte liegt; und b) die Existenz des Tempelberges wird dem Betrachter des Mosaiks unterschlagen. Der Cardo maximus und die davon am südlichen Ende nach Osten abgehende Nea sind auf der Madaba-Karte dagegen topographisch richtig wiedergegeben und betonen die Größe und Bedeutung der beiden justinianischen Bauprojekte. ${ }^{7}$

\section{II}

Kyrill von Skythopolis erzählt in seiner um 555 entstandenen Vita des Heiligen Sabas, wie der greise Wüstenvater 531 bei seinem Aufenthalt am Kaiserhof in Konstantinopel mehrere bedeutende Privilegien für die Kirche von Palästina erwirkte. ${ }^{8}$ Die Nea spielte bei den Verhandlungen angeblich eine wichtige Rolle, denn Kyrill zufolge bat Sabas den Kaiser, die Theotokos-Kirche in der heiligen Stadt zu bauen und auszustatten, deren Fundamente einige Zeit zuvor unter dem Jerusalemer Erzbischof Elias (494-515) gelegt worden seien. ${ }^{9}$ Mit großem Eifer, so Kyrill weiter, erfüllte der Kaiser die Bitte des Mönchsführers, stellte Geld zur Verfügung und sandte den Architekten Theodoros nach Jerusalem, um die Kirche zu bauen. Ohne die übergeordnete Autorität des Erzbischofs Petros anzutasten, beauftragte er den Bischof Barachos von Bakatha mit der Überwachung der Bauarbeiten, die nach zwölf Jahren mit der Einweihung zum Abschluß kamen. „Es ist überflüssig“, sagt Kyrill abschließend, „die Größe, den unglaublichen Glanz und die reiche Dekoration dieses ehrwürdigen Gebäudes zu beschreiben, denn es steht offen vor unseren Augen und übertrifft nicht nur alle Mo-

7 Vgl. Y. Tsafrir, The Holy City of Jerusalem in the Madaba Map, in: M. Piccirillo - E. Alliata (eds.), The Madaba Map Centenary (1897 - 1997). Travelling Through the Byzantine Umayyad Period, Jerusalem 1999, 155 -163, bes. 155-158; S. Mucznik - A. Ovadiah - Y. Turnheim, Art in Eretz Israel in Late Antiquity. Collectanea, Tel Aviv 2004, bes. 23. Zu Zweck und Bedeutung der Karte vgl. die einleuchtenden Überlegungen von I. Shahid, The Madaba Mosaic Map Revisited: Some New Observations, in: Piccirillo - Alliata (s.o.).

8 Kyr. Skyth. Vita Sabae 70 - 73 (171,26-178,18 Schwartz); vgl. Trampedach (s. Anm. 3) 279-284. F. Diekamp, Die origenistischen Streitigkeiten im sechsten Jahrhundert und das fünfte allgemeine Konzil, Münster 1899, 11-15, hat gezeigt, daß Kyrills Datierungen nach 529 ein Jahr hinzugefügt werden muß, so daß Sabas' Reise zu Justinian nach Konstantinopel ins Jahr 531 (statt 530) fällt. E. Stein, Cyrille de Scythopolis. A propos de la nouvelle édition de ses œvres, Analecta Bollandiana 62, 1944, 169 - 186, hier 171 - 180, hat dieses Ergebnis gegen die Einwände von E. Schwartz, Kyrillos von Skythopolis, Leipzig 1939, 343 - 346, verteidigt und mit zusätzlicher Evidenz versehen; vgl. auch L. Di Segni, Greek Dedicatory Inscription from the Vaulted Structure of the Nea Church, in: Gutfeld (s. Anm. 1) 259-267, hier 261 mit Anm. 4.

9 Kyr. Skyth. VS 72 (175,11-15). 
numente, die von uns gesehen wurden, sondern auch diejenigen, über die die Hellenen in ihren Geschichtswerken berichtet haben. “10

Kyrills Bericht setzt drei Akzente, um Justinians Leistung in die richtige Perspektive zu rücken: 1 . Der Kaiser sollte mit dem Bau der neuen Theotokos-Kirche in Jerusalem ein Werk fortführen und zu Ende bringen, das bereits Jahre zuvor von Patriarch Elias begonnen wurde. 2. Der Anstoß zu dem justinianischen Bau ging, wie Kyrill besonders betont, von der palästinischen Kirche und ihrem Abgesandten, dem Mönchsführer Sabas, aus. ${ }^{11}$ 3. Der Kaiser erfüllte das ihm angetragene Werk mit gro-

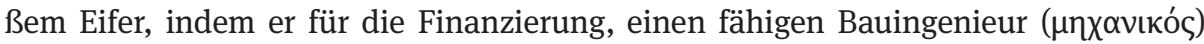
sowie geeignete Aufseher Sorge trug. Die Rollenverteilung, die Kyrill beschreibt, entspricht genau seiner Wunschvorstellung: Der Kaiser bewährt und profiliert sich als Helfer der Kirche von Jerusalem, die durch ihre Patriarchen und vor allem durch heilige Wüstenväter wie Sabas repräsentiert wird. ${ }^{12}$

Mit dem zitierten Urteil Kyrills stimmt Prokops Beschreibung, die ungefähr gleichzeitig entstand und den Kirchenbau als ein Wunder rühmt, überein. Im Unterschied zu Kyrill hielt Prokop glücklicherweise eine ausführliche Schilderung, die vor allem die Überwindung der enormen Schwierigkeiten bei der Errichtung der Kirche hervorhebt, nicht für überflüssig. ${ }^{13}$ Der unterschiedliche Fokus der beiden Werke erklärt sich aus den verschiedenen Genrezusammenhängen und Adressatenkreisen. Während Kyrill zur Verherrlichung der palästinischen Kirche und ihres Mönchtums schreibt, ${ }^{14}$ ist Prokops Werk originelle klassizistische Kaiserpanegyrik, die dem Leser anhand der kaiserlichen Baupolitik in Konstantinopel und den Provinzen die Nähe des Kaisers zu Gott möglichst anschaulich vor Augen zu führen versucht. ${ }^{15}$ Die Kaiserpanegyrik der „Bauten“ könnte erklären, warum Prokop in seiner Beschreibung der Nea weder eine Grundsteinlegung durch den Jerusalemer Patriarchen noch eine An-

10 Kyr. Skyth. VS 73 (177,14-178,4). Aus einer anderen Stelle seines Werkes (VE 49 [71,16-20]) geht hervor, daß Kyrill als junger Mönch an der Einweihung der Nea im November 543 persönlich teilgenommen hat.

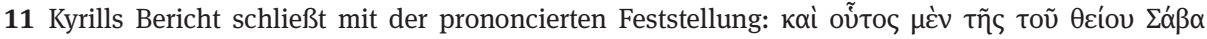

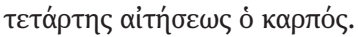

12 Dementsprechend fügt Sabas, so Kyrill VS $72(175,15)$, seiner Bitte an den Kaiser um die Voll-

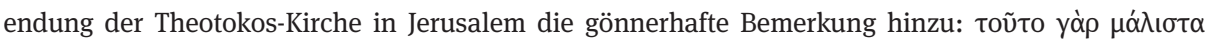

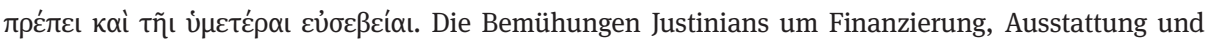
verantwortliches Personal erinnern an Konstantins Anordnungen zum Bau der Anastasis-Basilika: vgl. Eus. VC 3,31-32. Der mechanikós Theodoros, den der Kaiser von Konstantinopel nach Jerusalem schickte, ist wahrscheinlich derselbe, den Prokop (bell. 2,13,26) im Zusammenhang mit der erfolg-

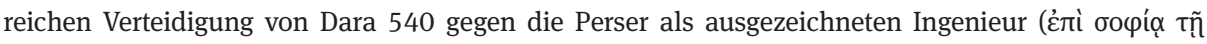

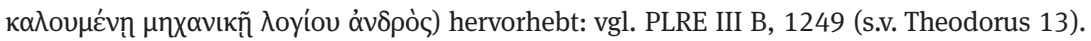

13 Prok. aed. 5,6.

14 Vgl. Trampedach (s. Anm. 3).

15 Vgl. Cameron (s. Anm. 2) 84-112; in der Datierungsfrage folge ich den Argumenten, die für etwa 554 als Abfassungsdatum vorgebracht wurden: Cameron (ebd.), 9-12; 85 f.; G. Greatrex, The Dates of Procopius Work, Byzantine and Modern Greek Studies 18, 1994, 101 -114; J. Howard-Johnston, The Education and Expertise of Procopius, Antiquité Tardive 8, 2000, 19-30, hier 20-22. 
regung durch den Wüstenvater noch andere mit der Durchführung des Baues betraute Funktionäre erwähnt, sondern den ganzen Ruhm des Unternehmens auf Justinian versammelt. Nach Prokop habe der Kaiser befohlen, die Kirche auf dem höchsten Hügel von Jerusalem zu errichten, nachdem er ihre sonstige Anlage sowie ihre Breite und Länge bestimmt hatte. ${ }^{16}$ Dies widerspricht der Darstellung Kyrills von der Grundsteinlegung durch den Patriarchen Elias. ${ }^{17}$ Doch könnte Kyrill diese Grundsteinlegung auch wenn nicht erfunden, so doch übertrieben haben, und ebenso könnte die Behauptung der Anregung durch Sabas eine hagiographische Zuspitzung sein. Schließlich führte Kyrill auch die späteren militärischen Erfolge Justinians in Afrika und Italien auf die Prophezeiungen und Gebete von Sabas zurück. Im Fall der Nea hatte Kyrill ein noch näher liegendes Interesse, seiner palästinischen Kirche und ihrem Mönchsführer die Initiative für das neue prächtige Bauwerk in Jerusalem zuzuschreiben. Ich werde auf das Problem zurückkommen.

Prokops Bericht über den Bau und die Gestalt der Kirche läßt sich gut mit den archäologischen Forschungen vereinbaren, die in den siebziger Jahren des 20. Jahrhunderts zur Aufdeckung von Substruktionen und Fundamentresten unter Leitung des israelischen Archäologen Nahman Avigad geführt haben. ${ }^{18}$ Dies gilt auch für den spektakulärsten Fund, den die Ausgräber in einer Zisterne machten, die unmittelbar an die südliche Stützmauer der Kirche angrenzte und durch eine gedeckte, mit Stufen versehene Galerie von Norden her zugänglich war. Auf der dem Eingang gegenüberliegenden Südseite fanden sie eine durch eine tabula ansata gerahmte Bauinschrift:

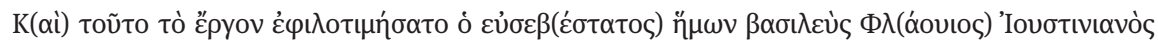

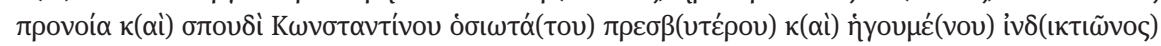
เу’ +

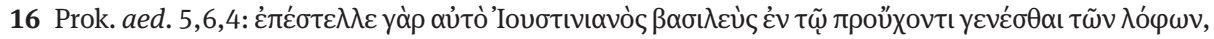

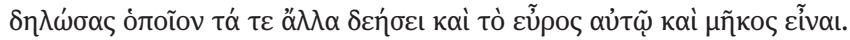

17 Merkwürdigerweise wurde dieser Widerspruch zwischen Kyrill und Prokop in der Forschungsliteratur zur Nea kaum zur Kenntnis genommen. Häufig werden die beiden Berichte als ergänzende Zeugnisse verstanden: vgl. K. Bieberstein - H. Bloedhorn, Jerusalem. Grundzüge der Baugeschichte vom Chalkolithikum bis zur Frühzeit der osmanischen Herrschaft, 3 Bde., Wiesbaden 1994, hier II 292; S. Shoemaker, Ancient Traditions of the Virgin Mary's Dormition and Assumption, Oxford 2002, $101 \mathrm{f}$.; M. Küchler, Jerusalem. Ein Handbuch und Studienreiseführer zur Heiligen Stadt, Göttingen 2007, 527; di Segni (s. Anm. 8) 259; Gutfeld (s. Anm. 1) 488f. Auf die unterschiedliche Akzentsetzung hingewiesen haben immerhin Y. Tsafrir, Procopius and the Nea Church in Jerusalem, Antiquité Tardive 8, 2000, 149-164, hier 151. 154; Shahid (s. Anm. 1) 377 n. 15.

18 Vgl. N. Avigad, Die Entdeckung der „Nea“ genannten Marienkirche in Jerusalem, Antike Welt 10, 1979, Heft 3, 31 - 35; N. Avigad, Discovering Jerusalem, Nashville 1983, 229-246; N. Avigad, The Nea: Justinian's Church of St. Mary, Mother of God, Discovered in the Old City of Jerusalem, in:Y.Tsafrir (ed.), Ancient Churches Revealed, Jerusalem 1993, 128-135; Tsafrir (s. Anm. 17); Gutfeld (s. Anm. 1), bes. $226-245$. 
„Auch dieses Werk förderte unser allerfrömmster Kaiser Flavius Justinianus freigiebig [oder rechnete sich ... zur Ehre an] unter der Fürsorge und mit dem Einsatz des heiligsten Presbyters und Hegoumenos Konstantinos im 13. Jahr der Indiktion.“"19

Das Werk, von dem hier gesprochen wird, ist natürlich die Zisterne, in der die Inschrift angebracht wurde. Die Inschrift blieb der Öffentlichkeit verborgen und kann allenfalls bei der Einweihung des Gebäudekomplexes gesehen worden sein. Unter der langen Regierung Justinians gab es ein 13. Indiktionsjahr dreimal: 534/5, 549/50 und 564/65. Da die Substruktionen, zu denen die Zisterne gehört, zweifellos vor dem eigentlichen Kirchenbau errichtet werden mußten, haben manche Forscher das erste Datum favorisiert. ${ }^{20}$ Dagegen spricht allerdings die Erwähnung des Presbyters und Hegoumenos Konstantinos, den auch Johannes Moschos als „Hegoumenos der heiligen Maria, der Theotokos, der Nea“ erwähnt. Es ist nämlich sehr unwahrscheinlich, daß es das Kloster bereits mehrere Jahre vor dem Heiligtum, zu dessen Betreuung es gegründet wurde, gab. Außerdem verweist das einleitende „auch“ anscheinend auf die bereits vollendete Nea und die anderen Bauten Justinians in Jerusalem. Am plausibelsten erscheint daher die Annahme von Leah di Segni, daß die gewaltigen Gewölbe auf der Südost-Seite des Nea-Komplexes unter dem Abt Konstantin 549/50 oder 564/65 zu einer unterirdischen Zisterne ausgebaut wurden und daß die Inschrift diesen Vorgang dokumentiert. $^{21}$

Die vier hauptsächlichen Schwierigkeiten, die nach Prokop mit dem Bau verbunden waren, lassen sich im archäologischen Befund gut nachvollziehen: 1. die Fundamente und Substruktionen, 2. der Transport der Baumaterialien, 3. die Dachkonstruktion und 4. die Säulen. Ich gehe diese Schwierigkeiten und ihre Überwindung der Reihe nach durch.

1. Prokop beschreibt die hügelige Topographie Jerusalems, den schroffen Wechsel von Berg und Tal, als besondere Herausforderung für die Bauherrn und Architekten. Die Hügelspitze sei eigentlich zu schmal für eine Kirche dieser Größe gewesen, daher habe man gewaltige Substruktionen an der Südost-Seite errichtet. Prokops Darstellung wird durch die Ausgrabungen bestätigt. Vor allem anderen haben sie eben diese

19 CIIP 1,800; vgl. jetzt di Segni (s. Anm. 8).

20 Z. B. Cameron (s. Anm. 2) 95; Amitzur (s. Anm. 1) 174; Muznik-Ovadiah-Turnheim (s. Anm. 7) 28; Küchler (s. Anm. 17) 527.

21 Di Segni (s. Anm. 8) 261f. Kurze Zeit nach der Einweihung der Nea berief Patriarch Petros den origenistischen Mönch und früheren Abt des Martyrios-Klosters, Johannes den Eunuchen, zum (offenbar ersten) Hegoumenos des zur Kirche gehörigen Klosters (Kyr. Skyth. VS 86 [193, 17 f.]). Vor dem Hintergrund des Origenistenstreits ist es gut möglich, daß dieser Johannes vor 549/50, sicher aber nach 554 durch den in der Inschrift genannten Konstantin abgelöst wurde. Von den im Pratum Spirituale des Johannes Moschos erwähnten Hegoumenoi des Nea-Klosters, Konstantinos (PG 87/3, p. 2857, cap. 6), Eudoxios (p. 3064, cap. 187) und Abramios (p. 2917, cap. 68; p. 3064, cap. 187), ist der erstgenannte vermutlich mit dem lokalen Bauherrn der Zisterne identisch. Auch in diesem wahrscheinlichen Fall wäre, wie di Segni zeigt, eine frühe Datierung nicht mit der internen Chronologie von Johannes Moschos zu vereinbaren. Vgl. auch Avigad, Discovering Jerusalem (s. Anm. 18) 134f.; D. Feissel, Les édifices de Justinien au témoignage de Procope et de l'épigraphie, Antiquité Tardive 8, 2000, 81-104, hier 99 f. 
aufwendige Fundamentierung ans Licht gebracht, die noch den heutigen Betrachter beeindrucken kann (2-7). Prokops panegyrische Übersteigerung entbehrt daher nicht einer Grundlage: „So steht das Bauwerk teils auf festem Felsengrund, teils schwebt es in der Höhe, nachdem des Kaisers Macht den Hügel erweitert hat“ (8).

2. Prokop hebt die Größe der verwendeten Steine hervor und die Schwierigkeiten des Transports (9-13). Auch diese Angaben werden durch die archäologischen Untersuchungen bestätigt. Die ausgegrabenen Blöcke im Bereich der Apsiden und der Südost-Ecke sind in der Tat - zumal für ein spätantikes Bauwerk - von bemerkenswerter Größe. ${ }^{22}$ Da sich die Steinbrüche vermutlich nördlich der spätantiken Stadt befunden haben, wird verständlich, warum die Straßenerweiterungen, von denen Prokop spricht, für den Bau der Nea schon allein aus logistischen Gründen notwendig wurden. Diese Maßnahmen können wir nach den Freilegungen und Restaurierungen der Archäologen heute besonders im südlichen Teil des Cardo noch gut nachvollziehen: Der Treppenweg, der hier wegen des hügeligen Geländes vermutlich vorher existierte, wurde durch eine Abtragung des Felses bis zu einer Höhe von 6 Metern eingeebnet und so verbreitert, daß Ochsenkarren die Steinquader für den Kirchenbau heranschaffen konnten. Außerdem wurde damit das Terrain für die repräsentative Säulenstraße bereitet, die überdies eine eindrucksvolle Verbindung von Nea und Anastasis schuf. ${ }^{23}$

3. Prokop entfaltet das Problem der Dachkonstruktion, verständlicherweise bei einer Kirche, deren Schiffe 74,6 m lang waren und bei der eine innere Breite von 52,3 m überbrückt werden mußte. Prokop erzählt von der schwierigen Suche nach genügend großen Bäumen; schließlich gelang es, so schreibt er, ein ausgedehntes Zedernwaldvorkommen zu erschließen, aus dem das Kirchendach in einer der Breite und Länge entsprechenden Höhe gezimmert werden konnte (14-15). Naturgemäß gibt es keinen archäologischer Befund, der beleuchten könnte, wie diese Herausforderung gelöst wurde.

4. Ein ähnliches Problem wie mit der Dachkonstruktion hatten die Baumeister nach Prokop hinsichtlich der Säulen (17-18). Säulen adäquater Größe und Stärke, um die Dachkonstruktion zu stützen und zu tragen, standen zunächst nicht zur Verfügung. Die Lage der Stadt im gebirgigen Binnenland verhinderte, so Prokop, den Import von Säulen, die gemäß dem spätantiken Usus monolithisch sein sollten. „Als sich jedoch der Kaiser“, sagt Prokop, „über die bauliche Schwierigkeit bedrückt fühlte, zeigte ihm Gott einen geeigneten Naturstein in den Nachbarbergen, wobei es fraglich bleibt, ob dieser zuvor schon dort verborgen lag oder sich damals erst bildete. In beiden Fällen kann man nämlich mit gutem Grund die Ursache auf Gott zurückführen; denn wir Menschen, die wir alles nach unserer schwachen Kraft bemessen, halten vieles für

22 Nach Tsafrir (s. Anm. 17) 154f., haben die Blöcke im Durchschnitt ein Gewicht von 4 Tonnen; einzelne Blöcke wiegen sogar mehr als doppelt so viel. M. Ben-Dov, In the Shadow of the Temple, Jerusalem 1985, 238, der die Südostecke unmittelbar jenseits der osmanischen Altstadtmauer ausgegraben hat, spricht (wohl ungenau) von Blöcken mit einem Gewicht von 5 bis 15 Tonnen.

23 Vgl. Tsafrir (s. Anm. 17) 155-162; Gutfeld (s. Anm. 1) 97-100. 484-487. 
unausführbar, Gott aber dürfte nichts von allem schwierig und unmöglich fallen. So stützt denn von dort eine große Zahl riesiger feuerfarbener Säulen allseits die Kirche, die einen unten, die anderen oben, andere wiederum rings die Säulenhallen, die abgesehen von der Ostseite das gesamte Heiligtum einschließen. Zwei davon, besonders hoch, so daß vielleicht keine größeren auf der ganzen Erde zu finden sind, stehen vor der Kirchentüre“ (19-22). Prokop fährt fort mit einer kurzen Beschreibung des Narthex, des Atriums, der Propyläen sowie zweier mit dem Baukomplex verbundener Hospize für Fremde und mittellose Kranke. Die Beschreibung endet mit der Bemerkung, daß der Kaiser die Kirche der Gottesmutter mit bedeutenden Einkünften ausstattete (23-26). ${ }^{24}$ Über die zuletzt genannten Teile des Baus läßt sich mangels archäologischer Evidenz kein klares Bild gewinnen. ${ }^{25}$ Anders verhält sich die Sache ausgerechnet im Fall der feuerfarbenen Säulen, an deren Gewinnung Prokop theologische Reflexionen der ihm eigenen Art anknüpft. Nach Yoram Tsafrir könnte es sich bei dem Rohstoff der Säulen um den harten, heute Mizzi Ahmar genannten Stein rötlicher Farbe handeln, der in Lagen nordwestlich, nördlich und nordöstlich der heutigen Altstadt von Jerusalem vorkommt. Prokops Bericht über die „wunderbare Entdeckung“ deutet nach Tsafrir darauf hin, daß dieser Stein von Justinians Steinmetzen zum ersten Mal in nennenswertem Umfang für Monumentalbauten genutzt wurde. Tatsächlich haben wir keine archäologische Evidenz für eine frühere Nutzung von Mizzi Ahmar in irgendeiner früheren Phase der Jerusalemer Baugeschichte. Wohl aber wenig später: Die fast 50 Säulen der wahrscheinlich unter Justinian renovierten Geburtskirche in Bethlehem stammen allem Anschein nach ebenfalls aus diesem Vorkommen. Trifft die Hypothese zu, so hätte der aufwändige Ausbau des Cardos nach Süden neben dem repräsentativen und symbolischen auch einen unmittelbar praktischen Sinn gehabt: als stufenlose Rampe, die es wesentlich erleichterte, die sehr langen Säulenschäfte von den Steinbrüchen im Norden zum Bauplatz zu bringen. ${ }^{26}$

24 Die Fremden- und Krankenhospize (sowie das Kloster) der Nea erwähnt auch der Pilger von Piacenza (Antonini Placentini Itinerarium, ed. C. Milani, Milano 1977, 23,1 p. 162 f.), der um 570 über seine Reise ins Heilige Land schrieb. In seinem Bericht über die Eroberung Jerusalems durch die Perser 614 nennt der Mönch Strategios unter den 35 Orten, an denen nach dem Abzug der Perser die Opfer des persischen Massakers aufgebahrt wurden (G. Garitte [ed. et transl.], Expugnationis Hierosolymae A.D. 614, Recensiones Arabicae I: A et B, 2 Bde. [arab. Text und lat. Übersetzung], CSCO 340 und 341, Louvain 1973, cap. XXIII, p. I 53 f. II 36 f. [A], I 102 f. II 68.f. [B]), die Nea (Nr. 5 der Liste), die Bibliothek der Nea (Nr. 8) und das kaiserliche Altersheim (Nr. 25): vgl. J.T. Milik, La Topographie de Jérusalem vers la fin de l'epoque byzantine, Mélanges de l'Université Saint Joseph de Beyrouth 37, 1960 - 61, 127 189, bes. $133.145-151$.

25 Eine mit vielen Abbildungen versehene Rekonstruktion, die den archäologischen Befund und die Beschreibung Prokops zusammenführt, versucht Gutfeld (Anm. 1) 226-245.

26 Tsafrir (s. Anm. 17) 162-164 (der im Übrigen vermutet [vgl. ebd. Anm. 35 und Fig. 13], daß die ca. 12 m lange monolithische Säule aus Mizzi Ahmar, die wegen eines Risses unvollendet im Steinbruch nahe der Russischen Kathedrale nordwestlich der Altstadt liegen blieb und dort heute in einer Bodenvertiefung betrachtet werden kann, für die Nea bestimmt war); vgl. Gutfeld (s. Anm. 1) 490f. Nicht überzeugend ist dagegen die (von Shahid [s. Anm. 1] 381 übernommene) Annahme von Ben-Dov (s. Anm. 22) 239f., Prokop wolle mit seiner Ausdrucksweise die Tatsache verschleiern, daß für die 
Im Folgenden möchte ich auf der Grundlage der bisherigen Ergebnisse die Frage erörtern, welche Motive zur Errichtung der Nea geführt haben. Doch zuvor muß ich auf die Frage der Urheberschaft zurückkommen. Es ist nach dem Gesagten m. E. klar, daß Kyrills Bericht trotz seiner scheinbar präzisen Angaben zumindest einen falschen Eindruck erweckt. Der Patriarch Elias kann die Fundamente zu einem Bauwerk dieser Größe an diesem Ort nicht gelegt haben. Dazu hätten ihm bei weitem die Mittel und die logistischen Voraussetzungen gefehlt. Denkbar wäre allenfalls, daß er mit dem Bau einer viel kleineren Marienkirche begann, wobei ungewiß bliebe, ob schon an dem Ort der späteren Nea oder anderswo in Jerusalem. Prokop stellt, wie gesagt, ausdrücklich fest, daß der Kaiser persönlich den Bauplatz, die Bauweise und die Ausdehnung der Kirche bestimmte. Diese Aussage ist zwar natürlich eine Übertreibung, aber kein Topos, denn einen solchen Anspruch erhebt Prokop an keiner anderen Stelle der Bauten. ${ }^{27}$ Selbst im Fall der Hagia Sophia in Konstantinopel äußert er sich zurückhaltender und verweist auf die Bedeutung der Fachleute, insbesondere auf die Architekten und Ingenieure Anthemios von Tralleis und Isidoros von Milet, die den Eifer des Kaisers unterstützten. Er fährt fort: „Es war aber auch dies ein Zeichen der Gnade Gottes für unseren Kaiser, daß er ihm die zu seinen Unternehmungen geeignetsten Männer an die Hand gab. Und auch die Klugheit des Kaisers selbst dürfte natürlich Bewunderung finden, deshalb weil er aus der ganzen Menschenschar die passendsten Helfer für die wichtigsten Werke auszuwählen verstand. “28 Trotz der allgemeinen Formulierung ignoriert Prokop diese Überlegung in seiner Beschreibung der Nea und

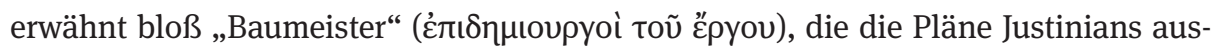
führten. Wie bei der Hagia Sophia kommen auch bei dem Bau der Nea die Klugheit des Kaisers und die Gnade Gottes zusammen, um das Werk gelingen zu lassen, bei der Nea allerdings eben ohne den Umweg über herausragende Mitarbeiter: „All dies schuf Kaiser Justinian mit menschlicher Kraft und Kunst. Doch trug zu diesem Unternehmen auch sein Gottvertrauen bei, das ihm die Ehre vergalt und bei diesem seinem Bemühen mitwirkte.“29 Diese panegyrische Zuspitzung an dieser Stelle ist m. E. kein leeres Gerede, sondern legt die Annahme nahe, daß Justinian sich tatsächlich selbst in einer nur mit der Hagia Sophia in Konstantinopel vergleichbaren Intensität mit dem Bau der Nea befaßt hat. Sehr wahrscheinlich hat er dabei auf die Mitarbeiter zurückgegriffen, die Kyrill nennt, insbesondere auf den Architekten und Bauingenieur Theodoros, den er

Errichtung der Nea die Ruine des jüdischen Tempels geplündert worden sei und daß insbesondere die Säulen von dort stammten. Die Säulen des herodianischen Tempels waren „nur“ 27 Fuß = 8,64 m hoch (d.h. kleiner als die der Nea) und wurden aus Trommeln zusammengesetzt: Jos. ant. 15,413; vgl. Avigad, Discovering Jerusalem (s. Anm. 18) 150-165.

27 Vgl. Amitzur (s. Anm. 1) $162 \mathrm{f}$.

28 Prok. aed. 1,1,25 f.

29 Prok. aed. 5,6,16. Es folgt der (oben zum Teil zitierte) Bericht über die wundersame Entdeckung des Steinbruchs, der den geeigneten Rohstoff für die Säulen der Kirchenschiffe und des Atriums bereithielt. 
aus Konstantinopel nach Jerusalem schickte. ${ }^{30}$ Weniger überzeugend erscheint dagegen die Darstellung über die Grundsteinlegung durch den Patriarchen Elias (494515), zumal die Frage offen bleibt, warum Elias' Nachfolger Johannes (515-524) und Petros (524-552) das Werk, das durch die Wirren, die durch die Interventionen des Kaisers Anastasios in der palästinischen Kirche entstanden, unterbrochen worden sein könnte, nach 518 nicht wieder aufgenommen haben. Wie dem auch sei: Da die angeblichen Fundamente des Elias jedenfalls über 15 Jahre lang liegen blieben und da das schließlich vom Kaiser realisierte Konzept die finanziellen und logistischen Möglichkeiten der Kirche von Jerusalem bei weitem überstieg, halte ich den Schluß für gerechtfertigt, daß Justinian eine ganz neue Nea baute, die mit der ursprünglichen Planung (wenn es sie überhaupt gab) nichts mehr zu tun hatte. Daher kann gefragt werden, welche symbolischen und theologischen Botschaften Kaiser Justinian mit dem Bau der Nea in Jerusalem vermitteln wollte.

\section{III}

Nach der Hagia Sophia in Konstantinopel war die Nea in Jerusalem der zweitgrößte und zweitteuerste Kirchenbau, den Justinian errichten ließ. In ihren Dimensionen und an architektonischem Aufwand übertraf sie auch den Neubau der Apostelkirche in Konstantinopel und die Johannes-Basilika in Ephesos. ${ }^{31}$ Darin kommt sicherlich die enorme Bedeutung zum Ausdruck, die Justinian Jerusalem einräumte. Doch welchen Spielraum gab es überhaupt um 530 für ein ambitioniertes Kirchenbauprojekt in Jerusalem? Die zentralen Schauplätze des Heilsgeschehens waren bereits besetzt. Die christliche Topographie Jerusalems im Jahre 531 weist Kirchen zur Erinnerung an das Oster- und Pfingstgeschehen, zur Erinnerung an die Wunderheilungen Jesu sowie über Märtyrergräbern und Reliquien auf. Die Nea dagegen läßt einen solchen Bezug vermissen; sie ist zur Zeit ihrer Errichtung die einzige namhafte Kirche in Jerusalem, die nicht auf, über oder an einem biblischen Erinnerungsort errichtet wurde. ${ }^{32}$ Daß ausgerechnet ein theologisch ambitionierter Kaiser wie Justinian in Jerusalem eine monumentale Kirche baute, die auf die heilsgeschichtliche Bedeutung des Ortes keinen Bezug nahm, darf wohl von vornherein als unwahrscheinlich gelten.

30 Über den Substruktionen der Südostseite, in die vermutlich später die erwähnte große Zisterne mit der Stiftungsinschrift eingebaut wurde (s.o.), haben die Ausgräber Mauern und Steinböden eines Gebäudes freigelegt, das vermutlich mit dem zur Nea gehörenden Kloster oder den von Prokop erwähnten Hospizen identifiziert werden kann. Diese Mauern bestehen aus alternierenden Schichten von Steinen und Ziegeln. Die Vermutung des Ausgräbers Nahman Avigad (Entdeckung [s. Anm. 18] 33; Discovering Jerusalem [s. Anm. 18] 236; vgl. Gutfeld [s. Anm. 1] 146) ist naheliegend, daß diese Technik, die in Konstantinopel sehr verbreitet, in Jerusalem dagegen bis dahin unbekannt war, von dem kaiserlichen Architekten Theodoros in Jerusalem eingeführt wurde.

31 Vergleiche mit anderen Kirchenbauten der justinianischen Zeit unternimmt auch: Gutfeld (s. Anm. 1) $487 \mathrm{f}$.

32 Vgl. die Skizze bei Bieberstein/Bloedhorn (s. Anm. 17) I 153-164. 


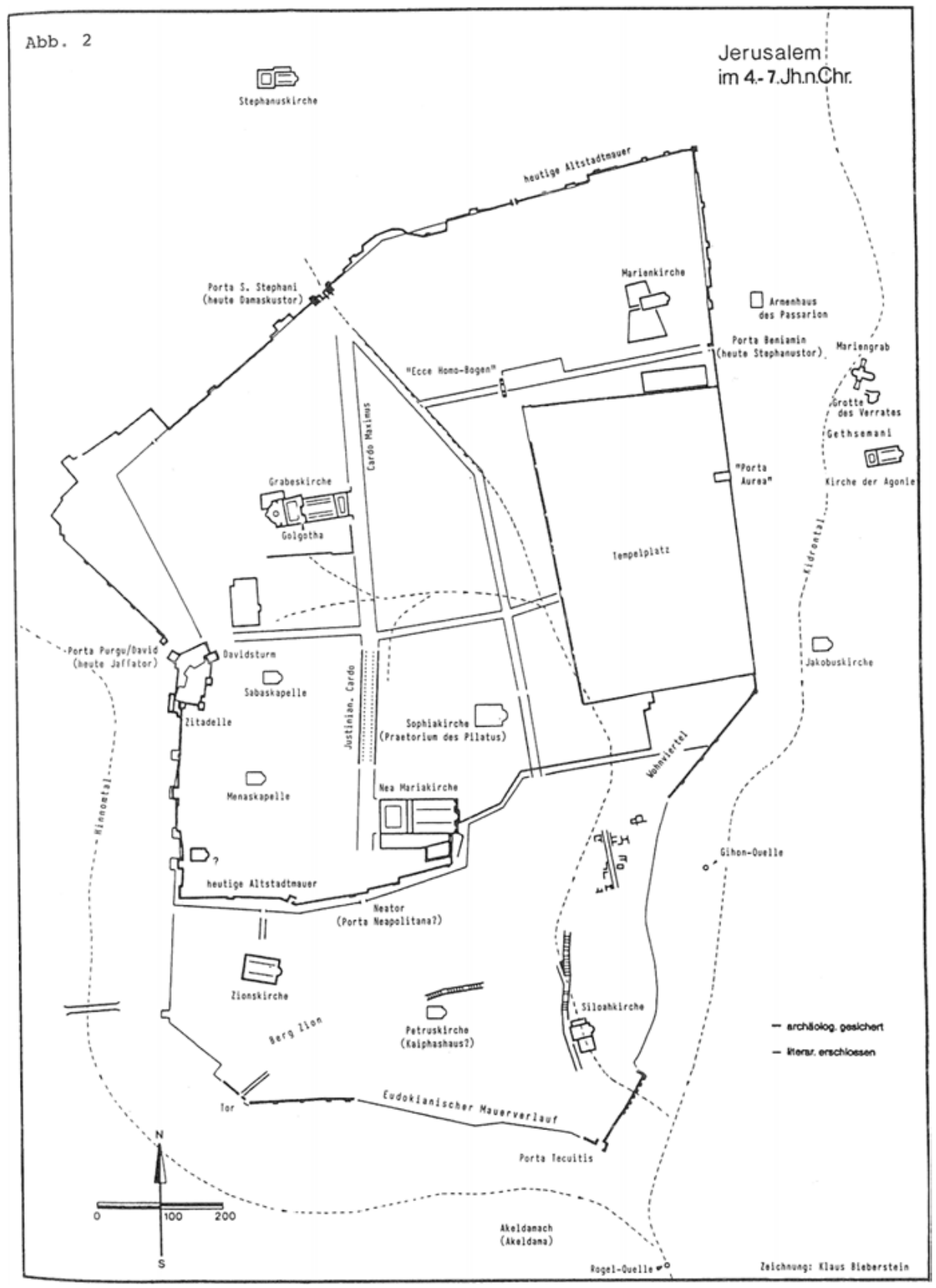

Abb. 1: Plan von Jerusalem im 4.-7. Jh. n. Chr., aus: RAC 17 (1996), $657 \mathrm{f}$.

Wie das historische Defizit ausgeglichen werden sollte, verrät ein Blick auf die physische Topographie von Jerusalem. Die Nea liegt auf etwa gleicher Höhe durch Täler getrennt vis-à-vis von Tempelberg und Anastasis-Komplex. Verbindet man die drei 
Punkte, so entsteht ein gleichschenkliges Dreieck. „Kaiser Justinian befahl, die Kirche auf dem höchsten Hügel zu errichten“, schreibt Prokop, ${ }^{33}$ und wir haben gesehen, daß er für dieses Ziel große Anstrengungen unternehmen mußte. Die herausgehobene Lage muß ihm also sehr wichtig gewesen sein, und natürlich war sie überdies geeignet, die Monumentalität des Bauwerks zu steigern. Darüber hinaus will der Kaiser seine Kirche offensichtlich durch ihre spezielle Position mit den beiden anderen herausragenden Orten in Jerusalem in Verbindung bringen: dem Anastasis-Komplex und dem jüdischen Tempelberg.

Nicht nur durch die Position auf dem gegenüberliegenden Hügel, sondern auch durch die Größe und Ausstattung konkurrierte die Nea mit dem konstantinischen Zentralheiligtum der Stadt. Andererseits schuf der Ausbau des Cardo eine direkte und repräsentative Verbindung zwischen den beiden monumentalen Kirchenkomplexen. Man kann das Verhältnis zwischen den beiden Bauten vielleicht so ausdrücken: Mit seiner Marien-Basilika stellte Kaiser Justinian Konstantins Christus-Kirche ein ähnlich ambitioniertes christliches Heiligtum als würdiges Gegenstück zur Seite. ${ }^{34}$ Denn übertroffen werden konnte der Ort des Leidens und der Auferstehung Christi natürlich durch keine Pracht der Welt.

Nach gut 250 Jahren der Bedeutungslosigkeit wurde Jerusalem durch Konstantin aufs Neue als heilsgeschichtlicher Ort etabliert. In der Vita Constantini spricht der Theologe Eusebios von Kaisareia von des Kaisers neuem Jerusalem, „gegenüber dem altberühmten, das nach der Befleckung durch die Ermordung des Herrn in die äußerste Verwüstung gestürzt worden war und so die Strafe für seine gottlosen Einwohner büßte“. ${ }^{35}$ Der Satz verrät das Problem, vor das sich Eusebios durch ein irdisches Jerusalem der Christen gestellt sah. Was ihm und anderen Theologen einst eines der wichtigsten Unterscheidungsmerkmale vom Judentum gewesen war, drohte zu verschwinden. Daher wurde die neue christliche Stadt, die um das heilige Grab gruppiert ist, von der alten jüdischen, von Gott verworfenen und auf den Tempelberg orientierten Stadt demonstrativ räumlich abgesetzt, blieb aber zugleich in der als Überwindung verstandenen Absetzung auf diese bezogen. Die Topographie legt die Annahme einer ähnlichen Dialektik auch für Justinians Kirche nahe, zumal diese den mit Ruinen bedeckten und als Müllplatz benutzten Tempelberg sichtbarer überragte als der Anastasis-Komplex. ${ }^{36}$

33 Prok. aed. 5,6,4.

34 Nach Küchler (s. Anm. 17) 529f. wirkt die Nea auf dem Madaba-Mosaik „wie ein Gegenstück zur Grabeskirche“: „Darin wird die ehrgeizige Absicht Justinians ersichtlich, am prachtvoll verlängerten Cardo maximus Konstantins die größte Marienkirche Palästinas zu errichten.“

35 Eus. VC 3,33,1 (Übers. H. Schneider, Turnhout 2007).

36 Zur Topographie s. Gutfeld (s. Anm. 1) 4-6 (mit Fig. 2). 141 sowie die Schlußfolgerung 491: „By emphasizing the construction of the church on the highest hill in the city, was Procopius referring to Justinian's intent to have it overlook the ruins of the Temple Mount - and thus underscore Christianity's superiority and victory over Judaism?" Vgl. auch Amitzur (s. Anm. 1) 171; A.S. Jacobs, Remains of the Jews. The Holy Land and Christian Empire in Late Antiquity, Stanford 2004, 151; Shahid (s. Anm. 1) $377 \mathrm{f}$. 
Im christlichen Jerusalem drängt sich die Frage nach dem Verhältnis zum jüdischen Tempel geradezu von selbst auf; sie ist aber auch in Konstantinopel zu dieser Zeit ein „heißes Eisen“. Anicia Juliana, eine reiche Aristokratin mit kaiserlichen Ahnen, ließ in Konstantinopel die bis dahin größte und prächtigste Kirche am Ort errichten, die, dem Märtyrer Polyeuktos geweiht, in den zwanziger Jahren des 6. Jahrhunderts vollendet wurde. Aus der Stiftungsinschrift, die mit vielen Worten in poetischer Sprache die Leistung und hohe Abstammung der Stifterin rühmt, geht hervor, daß Anicia Juliana ihre Polyeuktoskirche als Überbietung des salomonischen Tempels verstanden wissen wollte. ${ }^{37}$ Justinian begriff die Kirche und diesen Anspruch offenbar als Herausforderung, die er mit dem Neubau der Hagia Sophia beantwortete. Dementsprechend wurde die Hagia Sophia in Konstantinopel von der zeitgenössischen Überlieferung wiederholt mit dem salomonischen Tempel verglichen: In einem berühmten Hymnos hat Romanos Melodos den Wiederaufbau der Hagia Sophia in Konstantinopel durch Kaiser Justinian mit zwei Vorbildern aus Jerusalem verglichen, nämlich zum einen mit Salomon und dem Tempel und zum anderen mit Konstantin und Helena und dem Bau der Anastasis- und der Zionskirche. ${ }^{38}$ Wenn sich aber dieser Vergleich aus theologischen Gründen für die wichtigste und größte Kirche der Hauptstadt anbot, dann mußte das umso mehr für einen monumentalen Kirchenbau am eigenen Ort gelten. Und Justinian soll bekanntlich bei Einweihung der Hagia Sophia ausgerufen haben: „Ich habe dich übertroffen, Salomon!“39 Nun konnte Justinian im Jahre 531, als der Plan zum Bau der Nea entwickelt wurde, noch nicht wissen, daß er kurze Zeit später (als Folge der Zerstörungen des Nika-Aufstandes im Januar 532) Gelegenheit bekommen würde, die Hagia Sophia neu zu bauen. Daher dürfte ihm schon der Besuch des Wüstenvaters Sabas in Konstantinopel einen willkommenen

37 Anthologia Palatina 1,10,48-50; vgl. M. Harrison, Ein Tempel für Byzanz. Die Entdeckung und Ausgrabung von Anicia Julianas Palastkirche in Istanbul, Stuttgart - Zürich 1990, 137 - 139; J. Bardill, A New Temple for Byzantium: Anicia Juliana, King Solomon, and the Gilded Ceiling of the Church of St. Polyeuktos in Constantinople, in: W. Bowden - A. Gutteridge - C. Machado (eds.), Social and Political Life in Late Antiquity, Leiden 2006, 339 - 370. Daß über Jerusalem und den Tempel auch in der zweiten Hälfte des 6. Jahrhunderts in Konstantinopel debattiert wurde, ja daß sogar der Wiederaufbau des jüdischen Tempels denkbar erschien, bezeugen die Erotapokriseis des Pseudo-Kaisarios (ed. R. Riedinger, Berlin 1989) IV 218: vgl. Y. Papadoyannakis, A Debate about the Rebuilding of the Temple in Sixth-Century Byzantium, in: G. Gardner - K.L. Osterloh (eds.), Antiquity in Antiquity. Jewish and Christian Pasts in the Graeco-Roman World, Tübingen 2008, 373-382.

38 Rom. Mel. 54,21 - 22; vgl. Anonymi in Hagiam Sophiam Hymnus, in: C.A. Trypanis, Fourteen Early Byzantine Cantica, Wien 1968, Nr. 12, Str. 3 und 13 (p. 142 und 145); Coripp. In laudem Iustini Augusti minoris 4,283 mit dem Kommentar von Av. Cameron, London 1976, $204 \mathrm{f}$.

39 Script. Orig. Const. p. I 105,4- 5 Preger. Angesichts der zeitgenössischen Parallelzeugnisse sollte dieser Ausspruch trotz seiner Überlieferung in einer späten und unzuverlässigen Quelle „nicht voreilig als unhistorisch abgetan werden“, wie M. Meier, Das andere Zeitalter Justinians. Kontingenzerfahrung und Kontingenzbewältigung im 6. Jahrhundert n. Chr., Göttingen 2003, 189, betont. Zur Hagia Sophia und der damit verbundenen „Salomon“-Problematik vgl. zuletzt W. Brandes, Der Nika-Aufstand, Senatorenfamilien und Justinians Bauprogramm, in: M. Meier - S. Patzold (eds.), Chlodwigs Welt. Organisation von Herrschaft um 500, Stuttgart 2014, 239-265, hier bes. 244-247. 
Anlaß geboten haben, sein Ziel, den Tempel Salomons (und die Polyeuktoskirche Anicia Julianas) zu übertreffen, am heilsgeschichtlichen Ort, in Jerusalem, zu verwirklichen..$^{40}$ Auch aus der Perspektive Konstantinopels erscheint es daher plausibel, daß der Kaiser die Nea mit dem Ziel der Überbietung des jüdischen Tempels konzipierte.

Die Nea ist der Gottesmutter Maria geweiht. Kaiser Justinian hat, wie insbesondere Mischa Meier herausgearbeitet hat, die zunehmende Marienverehrung unter der Bevölkerung aufgegriffen und den Marienkult im ganzen Reich, vermutlich als eine Antwort auf die diversen Krisen der Zeit, stark gefördert. ${ }^{41}$ Einen Aspekt dieser Politik, den Kirchenbau, hat Prokop hervorgehoben: „Viele Kirchen hat Kaiser Justinian in allen Teilen des Römerreiches zu Ehren der Gottesmutter mit solchem Prunk, in solcher Größe und unter Einsatz derartig gewaltiger Mittel errichtet, daß schon die Betrachtung einer Schöpfung für sich zu der Annahme führen könnte, nur dieses einzige Werk sei von ihm ausgeführt worden und er habe in unermüdlicher Tätigkeit seine ganze Regierungszeit damit verbracht. “42 In diesem Rahmen spielt die Nea die Rolle eines Paradebeispiels.

Die „alten“ Marienkirchen Jerusalems an den Bethesda-Teichen und bei Gethsemane erinnerten an die Geburt Mariens, die am 8. September gefeiert wurde, und an ihr Elternhaus, über dem sie angeblich errichtet wurde, sowie an ihren Tod und ihr Grab. ${ }^{43}$ Die Nea dagegen ist, wie erwähnt, mit keinem biblischen Schauplatz unmittelbar verbunden. Eine Verbindung zum Heilsgeschehen konnte nur durch eine liturgische Einbindung in das Jerusalemer Festjahr hergestellt werden. Doch wie wurde das bewerkstelligt, wie wurde die Nea in das liturgische Kirchenjahr von Jerusalem eingegliedert? Der zeitliche Zusammenhang zwischen dem Datum der Kirchweihe, dem

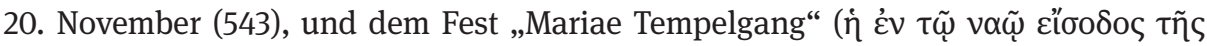

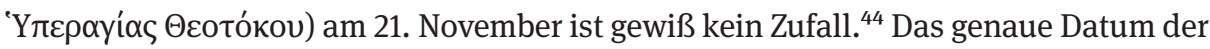
Aufnahme des Festes in die Jerusalemer Stationsliturgie ist unbekannt; die liturgiegeschichtliche Überlieferung verweist den Beginn des Festes in die Mitte des 6. Jahr-

40 Vgl. Amitzur (s. Anm. 1) 174 und bes. Shahid (der die kaiserliche Selbstdarstellung Justinians als „better-than-Solomon emperor“ [s. Anm. 1, 374. 376. 380. 385] hervorhebt).

41 Meier (s. Anm. 39) 502-528.

42 Prok. aed. 1,3,2; vgl. Meier (s. Anm. 39) 514 mit Anm. 448.

43 Vgl. Bieberstein/Bloedhorn (s. Anm. 17) III 167 - 169 (zu St. Maria in Piscina Probatica). III 251 256 (zur Kirche am Grab Mariens); Küchler (s. Anm. 17) 245 f. 470 f. Der Name „Nea“ wird in keiner zeitgenössischen Quelle erklärt. Nach der abwegigen Auffassung von K. Bieberstein, Die Porta Neapolitana, die Nea Maria und die Nea Sophia in der Neapolis von Jerusalem. Beobachtungen zur Stadtentwicklung in byzantinischer und früharabischer Zeit, ZDPV 105, 1989, 110-122 erhielt die Nea ihren Namen nicht in Abgrenzung zu einer früheren Marienkirche, sondern mit Bezug auf den Stadtteil Neapolis.

44 M. Tarchnischvili, Le Grand Lectionnaire de l'église de Jérusalem (Ve-VIIIe siècle), Tome I et II, Louvain 1959 - 1960, n. 1373; G. Garitte, Le Calendrier Palestino-Géorgien du Sinaiticus 34 (X siècle), Bruxelles 1958, 389. 391. Eine Verbindung der beiden Feste wird abgelehnt von S.C. Mimouni, Dormition et l'Assomption de Marie. Histoire des Traditions Anciennes, Paris 1995, 512- 514. 
hunderts. ${ }^{45}$ Offenbar knüpfte Mariae Tempelgang an die Kirchweihe an und fand auf diese Weise Eingang in den liturgischen Kalender Jerusalems (und verbreitete sich von dort zunächst in der Ostkirche, später auch im Westen). Das Fest erinnert an die Präsentation der dreijährigen Maria im Tempel und ihren neunjährigen Aufenthalt ebendort, ein Geschehen, das in dem apokryphen Protoevangelium des Jakobus geschildert wird. ${ }^{46}$ Worin liegt der Zusammenhang mit der Weihung der Nea? Nach Baldovin inspirierte wahrscheinlich die Nähe zu den Tempelruinen den Rückgriff auf Mariae Tempelgang. ${ }^{47}$ Doch läßt sich hier m. E. noch weiterkommen: Die Hymnen, die noch heute zu diesem Anlaß in der orthodoxen Kirche gesungen werden, feiern Maria

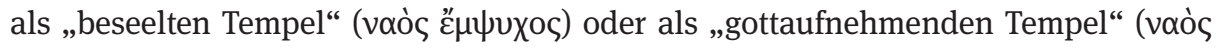

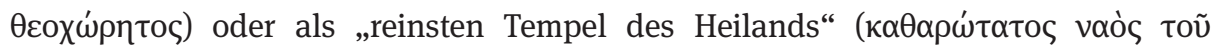
$\Sigma \omega \tau \tilde{n} \rho \circ) .{ }^{48}$ Dieser Sprachgebrauch, der offenbar auf Athanasios von Alexandreia zurückgeht, wurde besonders wirkmächtig von Johannes Chrysostomos und Kyrill von Alexandreia vertreten und ist seit dem Konzil von Ephesos geradezu konventionell. ${ }^{49}$ Im Zeitalter Justinians wird die Theotokos in zahlreichen Hymnen als vaò und mit ähnlichen Ausdrücken gerühmt. ${ }^{50}$ Nicht nur an Mariae Tempelgang, auch an anderen Tagen, in Zusammenhang mit großen Festen wie Epiphanien, Mariae Himmelfahrt und den Enkainien sowie zum Gedenken an die Erbauer Theodora und Justinian, war die Nea, wie aus dem georgischen Lektionar hervorgeht, Schauplatz der Jerusalemer Stationsliturgie. ${ }^{51}$ Von den Schriftpassagen, die an diesen Tagen in der

45 Vgl. J.F. Baldovin, The Urban Character of Christian Worship. The Origins, Development, and Meaning of Stational Liturgy, Roma 1987, 54. 239; Mimouni (s. Anm. 44) 376f.; Schoemaker (s. Anm. 17) 116 und - generell zur Geschichte der Jerusalemer Liturgie - H. Brakmann, RAC 17 (1996), $706-712$.

46 Protev. Jac. $7-8$.

47 J.F. Baldovin, Liturgy in Ancient Jerusalem, Bramcote 1989, 44.

48 MEГA $\Sigma$ IEPO $\Sigma$ ¿YNEK $\triangle$ EMO $\Sigma$, zum 21. November, p. $707-714$.

49 G.W.H. Lampe, A Patristic Greek Lexicon, Oxford 1961, s.v. vaós H. 2. Ungefähr zur gleichen Zeit wie

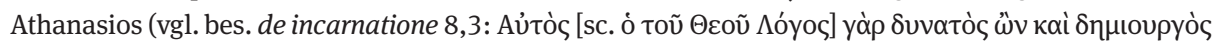

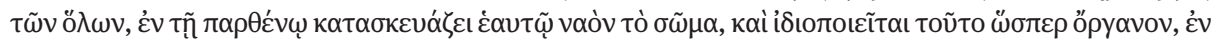

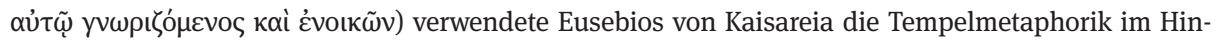
blick auf die Menschheit Christi, allerdings ohne den Zusammenhang mit der Jungfrau Maria zu erwähnen, und bezeichnenderweise anläßlich der Einweihung der Grabeskirche in Jerusalem (laud.

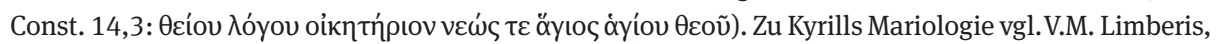
Divine Heiress. The Virgin Mary and the Creation of Christian Constantinople, London 1994, 107 - 116, bes. $109 \mathrm{f}$.

50 Rom. Mel. 35, Str. 2 und 5 (Maas/Trypanis p. 277 f.); Trypanis (s. Anm. 38) Nr. 1 (AkathistosHymnos), Str. 23 (p. 39); Nr. 11 (Auf die Aufnahme der heiligen Jungfrau Maria), Str. 19 (p. 137); Nr. 12 (Zur Einweihung der Hagia Sophia), Str. 3 (p. 142).

51 Am 4. Tag der Epiphanien-Oktav: Tarchnischvili (s. Anm. 44) n. 124. 16. März: Tarchnischvili, n. 258; vgl. Garitte (s. Anm. 44) 178. 26. Juni: Tarchnischvili, n. 1063; vgl. Garitte, 260. 3. August: Tarchnischvili, n. 1123; vgl. Garitte, 293. 17. August: Garitte, 305. 12. September: Tarchnischvili, n. 1232; vgl. Garitte, 328. Am 4. Tag der Enkainien-Oktav: Tarchnischvili, n. 1251; vgl. Garitte, 331. Zum georgischen Lektionar vgl. Baldovin (s. Anm. 45) 72-80; die Entwicklung der Marienliturgie in Jerusalem erörtert Schoemaker (s. Anm. 17) 132-141. 
Nea gelesen oder gesungen wurden, verweisen zumindest diejenigen für die Kirchweihe am 20. November und für den vierten Tag der Enkainia auf den Tempel. ${ }^{52}$ Wir können festhalten: Die Nea überragt den salomonischen Tempel nicht nur physisch; indem sie, wie ihre liturgische Integration in das Kirchenjahr deutlich macht, der Theotokos als dem wahren, dem eigentlichen nicht von Menschenhand gemachten Tempel Gottes geweiht ist, übertrifft sie ihn auch theologisch. Damit ist die Nea nicht nur die neue Marienkirche Jerusalems, sondern sie ist in gewisser Weise auch der neue, bessere Tempel.

Für diese Annahme kann man sich allerdings nicht auf den Autor Prokop berufen, wie dies etwa Oren Gutfeld tut, der in dem abschließenden Kapitel der Grabungspublikation (Chapter Twenty-Three: Discussion and Summary) das Verhältnis zwischen der Nea-Kirche und dem salomonischen Tempel erörtert. ${ }^{53}$ Weil Gutfeld dabei nicht zwischen dem Autor des Textes und dem Urheber des Bauwerkes, das durch den Text beschrieben wird, unterscheidet, traut er Prokop erstaunliche theologische Kenntnisse zu: „It seems that Procopius was also hinting at the prophet Isaiah's vision of the End of Days: ,In the last days the mountain of the Lord's temple will be established as the highest of the mountains; it will be exalted above the hills, and all nations will stream to it. (Isaiah 2:2). “54 Doch diese Aussage widerspricht auf eklatante Weise dem intellektuellen Profil eines klassizistischen Panegyrikers, der sich mehr für die Technik, Logistik und Ingenieurskunst der von ihm beschriebenen Bauten interessierte als für theologische Feinheiten. ${ }^{55}$ Nichts deutet außerdem darauf hin, daß Prokop über eine theologische Schriftgelehrsamkeit verfügte, die es ihm erlaubt hätte, subtile Anspielungen auf alttestamentliche Propheten zu machen. Wenn Prokop sich, was selten geschieht, auf die heiligen Schriften bezieht, tut er es offen, meist verkürzend und manchmal unrichtig. ${ }^{56}$

52 Zum 20. November: Ps. 131 (David gelobt den Tempelbau); Hebr. 3,1-6 (Christus ist höher als Moses); zum 4. Tag nach Enkainia vgl. H. Leeb, Die Gesänge im Gemeindegottesdienst von Jerusalem (vom 5. bis 8. Jahrhundert), Wien 1970, 77. 96.

53 Gutfeld (s. Anm. 1) 491-493, hier 491: ,it is hard to discern the great similarities between Procopius' description of the Nea Church and biblical descriptions of Solomon's Temple“.

54 Gutfeld (s. Anm. 1) 491; vgl. Taylor (s. Anm. 1) 52, die vage formuliert, daß die Position auf dem höchtsten Hügel von Jerusalem „recalls Isaiah 2:2“.

55 Howard-Johnston (s. Anm. 15), bes. 29, bescheinigt Prokop ein so ausgeprägtes Interesse an Baumaterial und Bautechnik, daß er ihn zuerst und vor allem zu einem Architekten/Ingenieur erklärt: „the expertise which he brought to Belisarius' staff was technical rather than literary or legal or organisational“.

56 Vgl. B. Rubin, RE 23 (1957), 341 - 343 (s.v. Prokopios von Kaisareia). Alttestamentliches kommt in den Bauten nur weniges und unspezifisches vor: Prokop erwähnt die babylonische Gefangenschaft der Judäer, um den Namen des nordsyrischen Städtchens Kyros zu erklären $(2,11,2)$. Er berichtet, daß Moses auf dem Berg Sinai die Gebote von Gott empfangen und dann den Menschen mitgeteilt haben soll $(5,8,8)$, ohne auf weitere mit dem Ort verbundene alttestamentliche Geschichten (wie z. B. die Legende vom brennenden Dornbusch) einzugehen. Sogar einen salomonischen Tempel nennt Prokop, aber nicht im Zusammenhang mit Jerusalem, sondern mit dem libyschen Ort Boreion; dort, so sagt er, „hatten die Juden einen alten Tempel, den sie ganz besonders in Ehren hielten, da nach ihren Worten 
Auch die Entdeckung des Zedernwaldes bei Prokop assoziiert Gutfeld mit der Erzählung des Königsbuchs über die „Zedern des Libanon“, die der König Hiram von Tyros schlagen ließ und dem König Salomon für den Tempelbau sandte. ${ }^{57}$ Abgesehen von der wenig aussagekräftigen Tatsache, daß in beiden Fällen Zedern zum Bau des Heiligtums verwendet wurden, sind jedoch keine Ähnlichkeiten zwischen den Texten zu erkennen; noch nicht einmal der Libanon findet bei Prokop Erwähnung. Wiederum gibt es nicht das geringste Anzeichen, daß Prokop den alttestamentlichen Text kannte oder im Sinn hatte; vielmehr ergibt sich aus seiner Darstellung mit wünschenswerter Klarheit, warum die Entdeckung des Zedernwaldes für die ungewöhnlich breite Dachkonstruktion und damit für die Vollendung des Bauwerkes von größter Bedeutung war.

Vielmehr deutet eine andere Bemerkung Prokops ein salomonisches Motiv an, das ihm selbst freilich unbewußt blieb. Der Panegyriker spricht von zwei besonders hohen und in ihrer Größe einzigartigen Säulen vor dem Eingang in die Basilika, ohne ihre Funktion oder Bedeutung zu erklären. Diese Leerstelle haben einige Forscher durch den Verweis auf eine Analogie im Alten Testament gefüllt: ${ }^{58}$ Nach 3 Kgt 7,1-9 (LXX) ließ König Salomon zwei prächtige Säulen, die Jachum und Baaz (bzw. Jachin und Boaz im MT) genannt wurden, an der Vorhalle des Tempelhauptraums errichten. Leider ist es nach Prokops Beschreibung nicht möglich zu verstehen, wo genau im Nea-Komplex die möglicherweise analogen justinianischen Säulen standen. Wie ist die Ortsangabe

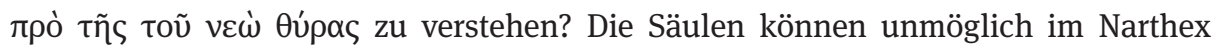
aufgestellt worden sein, den Prokop als nächstes erwähnt, denn, wie Gutfeld bemerkt, „the narthex was particularly narrow and such massive columns could not have been properly viewed, and certainly not properly appreciated by visitors. In addition, they would not have allowed for the presence of a gallery above the narthex, as required by Procopius' account.“ In ihrer Verlegenheit nehmen die Ausgräber Zuflucht zu einer Lösung, die m. E. auch nicht richtig befriedigt: „With all the difficulties it entails, we propose that the two columns stood on either side of the central apse, and that Procopius' description began in the east while facing west, his back towards the apse. First he surveyed the basilica, then turned back towards the apse and described the two massive columns, after which he proceeded westward to the narthex and atrium." 59

\footnotetext{
Salomon, der König der Hebräer, ihn erbaut haben soll“ (6,2,22). Direkte LXX-Zitate oder -Paraphrasen gibt es bei Prokop nicht. Hat Justinian bei der Errichtung der Nea vielleicht an Esaias 2:2 oder gar - wie Amitzur (s. Anm. 1) 166f. erwägt - an Ezechiel 40 gedacht? Dafür fehlt jeder positive Hinweis; in den Esaia-Kommentaren von Eusebios von Kaisareia (PG 24, 101-105), Johannes Chrysostomos (PG 56, 28f.) und Kyrill von Alexandrien (PG 70, 67-72) wird der Vers als Metapher verstanden und auf die Kirche im geistigen Sinne bezogen.

573 Kgt (LXX) 4,33 (5,13); Gutfeld (s. Anm. 1) 492; vgl. Shahid (s. Anm. 1) 377.

58 Amitzur (s. Anm. 1) 166; Shahid (s. Anm. 1) 377; Taylor (s. Anm. 1) 52; Gutfeld (s. Anm. 1) 243 f. (mit Fig. 5.23). 492f.

59 Gutfeld (s. Anm. 1) 243. Die Ausgräber glauben, in der Ostmauer auf der Nordseite der Apsis den Fundamentstein für eine monumentale Säule gefunden zu haben, und rekonstruieren einen Säulendurchmesser von 2,40 m bei einer Höhe von 17,75 m (einschließlich Basis und Kapitell).
} 
Diese Rekonstruktion widerspricht nicht nur dem Wortlaut Prokops; sie reduziert außerdem die Analogie der Nea-Säulen mit Jachin und Boaz, die demnach nicht mehr wie beim salomonischen Tempel vor dem Heiligtum stehen. Nicht erwogen haben die Ausgräber die Möglichkeit, daß die Säulen in dem an den Narthex anschließenden Atrium oder vor dem Eingang ins Atrium standen. Mangels Vergleichsbeispielen erscheinen allerdings auch diese Vorstellungen schwierig. So läßt sich die Frage nur konditional beantworten: Sollte Justinian mit den beiden Riesensäulen vor oder in der Nea versucht haben, Salomons Säulen vor dem Tempel nachzuahmen, hätte er einen Traditionsstrang wiederbelebt, der seine Kirche in eine besondere Gottesnähe rücken und ihre fehlende Verankerung in der neutestamentlichen Heilsgeschichte Jerusalems kompensieren konnte.

Ebenso rätselhaft wie die genaue Position der beiden Riesensäulen ist eine andere berühmte Angabe Prokops. In seiner Geschichte des Vandalenkrieges berichtet der Historiker, daß bei dem sogenannten Vandalentriumph Belisars 534 der Judenschatz, den Titus 70 nach der Einnahme Jerusalems mit anderen Beutestücken nach Rom gebracht hatte und der 455 von den Vandalen nach Karthago entführt worden war, durch die Straßen Konstantinopels in den Hippodrom getragen wurde. Prokop fügt hinzu, daß der Kaiser den Schatz anschließend dem Rat eines Juden folgend aus Angst

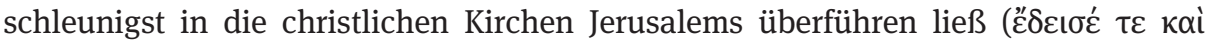

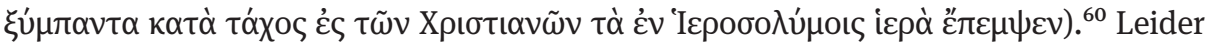
ist diese unpräzise Bemerkung Prokops das letzte, was über den jüdischen Tempelschatz überliefert wurde.

Der Vorgang erscheint schon deshalb merkwürdig, weil sich spätantike Kaiser gewöhnlich keine Gedanken um die Repatriierung von heiligen Gegenständen machten, sondern im Gegenteil große Anstrengungen unternahmen, um Reliquien aus allen Teil des Reiches, ganz besonders natürlich aus dem Heiligen Land, für ihre Hauptstadt Konstantinopel und ihren Palast zu erwerben. Folgt man Prokop, liegt die Vermutung nahe, daß Justinian die Gegenstände zur Aufbewahrung und Ausstellung in der Nea, seinem neuen Tempel in Jerusalem, bestimmte. ${ }^{61}$ Doch sprechen mehrere Indizien gegen die Glaubwürdigkeit von Prokops Bericht: ${ }^{62}$ In seiner Erzählung der Plünderung Roms durch Geiserich erwähnt Prokop nämlich den Judenschatz nicht. Stattdessen erwähnt er an anderer Stelle, daß Alarich „die sehenswerten Geräte des Hebräerkönigs Salomon, die einst die Römer aus Jerusalem mitgenommen hatten, darunter ein durch besondere Schönheit ausgezeichneter Smaragd“, bei der Einnahme Roms 410 geraubt und nach Carcasso ins südliche Gallien gebracht habe (bell. 5,12,4142), ${ }^{63}$ ohne den Widerspruch zu kommentieren. Auch andere Quellen, die früher und

60 Prok. bell. 4,9,5-9.

61 Taylor (s. Anm. 1).

62 Vgl. R.S. Boustan, The Spoils of the Jerusalem Temple at Rome and Constantinople: Jewish CounterGeography in a Christianizing Empire, in: Gardner - Osterloh (s. Anm. 37) 327-372, hier 356-362.

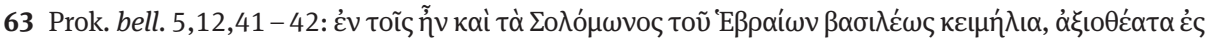

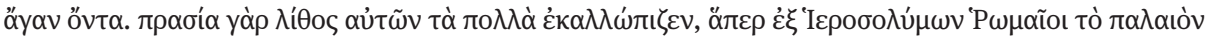


unabhängig von Prokop über die Plünderung Roms durch Geiserich berichten, wie Jordanes oder Victor von Vita, wissen offenbar nichts von den кعıй $\lambda \iota \alpha$ Salomons. Vollkommen unglaubwürdig ist außerdem die Art und Weise, wie Prokop die Weiterleitung der Tempelobjekte nach Jerusalem motiviert. Ausgerechnet ein Jude soll den Kaiser (über einen vornehmen Mittelsmann aus dessen Umgebung) veranlaßt haben, die aus dem Tempel stammenden Kostbarkeiten nach Jerusalem (,an den Ort, wo sie der Judenkönig Salomon früher aufstellen ließ“) zurückzugeben. Der Jude erreichte dies nach Prokop, indem er Justinian in die Angst versetzte, sein Palast in Konstantinopel könnte wegen der Anwesenheit des jüdischen Tempelschatzes das gleiche Schicksal wie die Paläste in Rom und Karthago erleiden. Doch wie ließe sich erklären, daß diese Objekte im Zeichen des orthodoxen Christentums noch eine Gefahr darstellten? Sollte Prokop hier seinen eigenen Aberglauben auf den Kaiser übertragen haben ${ }^{64}$ Nach Shahid, der Prokops Bericht mit zwei weiteren Argumenten zu stützen versucht, ,the existence of the Vessels in Constantinople would have been grist to the mill of the late Anicia Juliana, especially if they had been deposited in the Church of Polyeuktos, thus endowing it with an unusual sanctity“. Doch warum hätte Justinian die Gefäße der Polyeuktoskirche überlassen sollen? Er hätte sie ohne weiteres vorübergehend in einer Palastkirche aufbewahren können, um sie dann 537 der Hagia Sophia zu stiften. Shahids zweites Argument ist auch nicht plausibler: „The superstitious emperor may have remembered from reading the book of Daniel in the Old Testament that the misuse to which the vessels had been put by the Neo-Babylonian king Belshazzar finally led to the destruction of his kingdom. After he used the Vessels at his banquet, the moving finger appeared on the wall and prophesied his downfall, in the four mysterious words that Daniel interpreted for him. “65 Doch warum sollte sich der orthodoxe Kaiser Justinian mit dem heidnischen König Baltasar (Dan 5,1-5 LXX) identifizieren? Abgesehen von dessen blasphemischen Umgang mit den Tempelgefäßen würde eine solche Deutung die absurde Annahme voraussetzen, daß sich Justinian in seiner Funktion als Schutzherr der orthodoxen Christenheit nicht für den

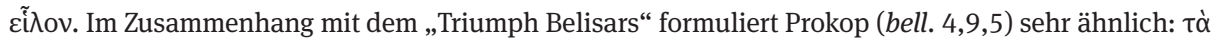

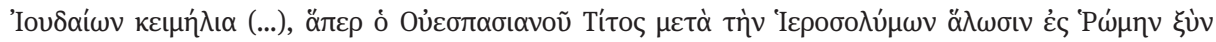

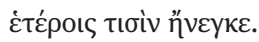

64 Warum aber sollte Prokop diese merkwürdige Geschichte erfunden haben? Boustan (s. Anm. 62) 360, gibt folgende Antwort: „It offers Procopius an effective rhetorical strategy for linking the triumph to the glorious victories of the Flavians over an earlier 'internal enemy' - in their case, the Jews rather than the Vandals - but without actually having to contend with the inconvenient traces that the vessels might have left behind in the capital." Diese Erklärung wird plausibler, wenn man von einer weitgehenden Übertreibung Prokops (statt von einer Erfindung) ausgeht. Vielleicht vermeidet es Prokop deswegen, die Gegenstände beim Namen zu nennen. Es ist jedenfalls schwer vorstellbar, daß er, der im Fall von Carcasso einen Smaragd der gesonderten Erwähnung für wert hielt (s. o.), spektakuläre Objekte wie den siebenarmigen Leuchter oder den goldenen Schaubrottisch verschwiegen hätte, wenn sie mit Belisar im Triumph durch Konstantinopel getragen worden wären.
}

65 Shahid (s. Anm. 1) $375 \mathrm{f}$. 
rechtmäßigen Besitzer der Tempelgefäße hielt. Nach allem, was wir über das religiöse Selbstverständnis Justinians wissen, muß das Gegenteil der Fall gewesen sein.

Wenn Belisar also überhaupt Gegenstände des Judenschatzes von Karthago nach Konstantinopel brachte und die ganze Geschichte von Prokop nicht erfunden, sondern nur übertrieben wurde, und wenn Justinian diese Gegenstände nach Jerusalem schickte, dann sicher nicht aus religiöser Furcht, sondern um seiner Vorstellung von der Überwindung des Judentums durch das Christentum am Ort der Erscheinung, des Leidens und der Wiederauferstehung Christi anschaulichen Ausdruck zu verleihen. Ein solches Kalkül würde allerdings voraussetzen, daß die Gegenstände aus dem salomonischen (eigentlich herodianischen) Tempel einer breiten christlichen Öffentlichkeit präsentiert werden sollten. Die Nea, an der 534 bereits drei Jahre gebaut wurde, wäre eine ideale Bühne für diesen Zweck gewesen. Doch hören wir nichts davon. Mangels Zeugnissen muß daher offen bleiben, ob der Judenschatz jemals in dieser Kirche oder an einem anderen Ort in Jerusalem angekommen ist. ${ }^{66}$

Leider verliert sich die Spur des vollendeten Bauwerks rasch im Dunkel der Geschichte. Der Pilger von Piacenza, der um 570 ins Heilige Land reiste, und Johannes Moschos am Anfang des 7. Jahrhunderts erwähnen die Nea. ${ }^{67}$ Auch in einer Mosaikinschrift in Jericho, die das Grab eines am 11. Dezember 566 gestorbenen Kyriakos schmückt, wird die Nea genannt. ${ }^{68}$ Bei der Eroberung Jerusalems durch die Perser im Jahre 614 wurde die Kirche offenbar beschädigt, aber nicht zerstört. ${ }^{69}$ Es gibt vereinzelte Hinweise, die auf eine Kultkontinuität bis zum frühen 9. Jahrhundert hindeuten, möglicherweise in einem kleineren Teil des riesigen Baukomplexes. ${ }^{70}$ Das in das Jahr $808 \mathrm{zu}$ datierende Commemoratorium de casis dei listet unter anderem eine vom Erdbeben beschädigte Nea auf und ordnet ihr einen Klerus von 12 Personen zu. ${ }^{71}$ Dagegen stellt Eutychios von Alexandria um 935 fest, daß die Nea 614 zerstört und danach nicht wieder errichtet wurde. ${ }^{72}$ Auch wenn diese Aussage wegen der bis ins

66 Taylor (s. Anm. 1) 54, glaubt dagegen, daß der Schatz in Jerusalem ankam, aber versteckt wurde: „The absence of any reference to the Temple treasure in the few accounts we do have may be due to the fact that it was not on show but stored below in the vaults, guarded by the monks and by the apotropaic care of the Mother of God.“

67 Antonini Placentini Itinerarium (s. Anm. 24) cap. 23; Joh. Mosch. cap. 6. 61. 68. 187 (PG 87/3, p. 2857. 2913. 2917. 3064; s. Anm. 21); vgl. Küchler (s. Anm. 17) 530-532.

68 SEG 8,315; Milik (s. Anm. 24) 147; Gutfeld (s. Anm. 1) 250.

69 Vgl. G. Avni, The Persian Conquest of Jerusalem (614 C.E.) - An Archaeological Assessment, BASOR 357, 2010, 35-48; Y. Stoyanov, Defenders and Enemies of the True Cross. The Sasanian Conquest of Jerusalem in 614 and Byzantine Ideology of Anti-Persion Warfare, Wien 2011, 11-23, bes. 19; G.W. Bowersock, Empires in Collision in Late Antiquity, Waltham 2012, 31 - 51.

70 R. Schick, The Christian Communities of Palestine from Byzantine to Islamic Rule. A Historical and Archaeological Study, Princeton 1995, $332 \mathrm{f}$.

71 Vgl. M. McCormick, Charlemagne's Survey of the Holy Land. Wealth, Personnel, and Buildings of a Mediterranean Church between Antiquity and the Middle Ages, Washington D.C. 2011, $103-111$. 72 M. Breydy, Das Annalenwerk des Eutychios von Alexandrien. Ausgewählte Geschichten und Legenden kompiliert von Sa’id ibn Batriq um 935 A.D., 2 Bde. (arab. Text und dt. Übersetzung), CSCO 471 und 472, Leuven 1985, hier I $98 \mathrm{f}$. II $118 \mathrm{f}$. 
frühe 9. Jahrhundert fortdauernden Erwähnungen der Nea in den Quellen sowie wegen der fehlenden archäologischen Evidenz nicht glaubwürdig ist, deutet sie zumindest darauf hin, daß die Nea bereits im 10. Jahrhundert weitgehend von der Bildfläche verschwunden ist. Dieser Untergang ist bemerkenswert - ein einmaliger Vorgang, der nach einer Erklärung verlangt: Weil die Nea nicht an einen Erinnerungsort im engeren Sinne gebunden war, sondern ihre Bedeutung einem artifiziellen theologischen Konzept verdankte, konnte sie seit dem 9. Jahrhundert trotz ihrer einstigen Monumentalität und liturgischen Verankerung sang- und klanglos aus der Sakraltopographie Jerusalems verschwinden. Außerdem verlor dieses theologische Konzept seine Pointe - es wurde sozusagen historisch „erledigt“ -, als das Tempelplateau nicht mehr Ruinenfeld war, als Müllkippe diente und auf diese negative Weise auf den zerstörten jüdischen Tempel verwies, sondern durch den Bau des Felsendoms und der Al-AqsaMoschee um die Wende vom 7. zum 8. Jahrhundert religiöses Zentrum des islamischen Jerusalem wurde. 
